\title{
Species of Septobasidium from Japan
}

By

\author{
Wataro YAMAMOTO*
}

In the recent work of Dr. S. Ito, "Mycological Flora of Japan", vol. II, no. 4, issued in 1955, there are listed six species of the genus Septobasidium as being recorded from Japan. They are Septobasidium bogoriense Pat., S. Tanakae (Miyabe) Boedijn et Steinm., S. prunophilum Couch, S. indigophorum Couch, S. mariani Bres. var. japonicum Couch and S. pilosum Boedijn et Steinm. Among them, the former two are very commonly found on various plants in Japan, and had long been called S. pedicellatum (Schw.) Pat. and Helicobasidium Tanakae Miyabe respectively. Among the other four species, the three species (including one variety) named by Couch were originally collected in Japan and described by him in his "Genus Septobasidium" in 1938.

During these years, the writer has collected many specimens of the genus at various localities in the provinces of Tamba, Settu, Kawachi, Yamasiro and Yamato. They are classified into six species, S. Tanakae, S. bogoriense and four new species, by the writer. In the present paper, the writer gives diagnoses of these species, and consequent upon his observations, he came to the conclusion that $\mathrm{S}$. prunophilum is identical with $\mathrm{S}$. Tanakae, and S. indigophorum is synonymous with $\mathrm{S}$. bogoriense. So, there exist nine species and one variety of the genus in the present Japanese mycological flora.

Key to the six species of Septobasidium

Basidià 2-celled.........1. S. nigrum YАMAMOTO

Basidia 4-celled

Basidia with probasidial cells

Basidia straight

Middle regions of context thin and not stratose......2. S. tambaensis YAMAMOTO

Middle regions of context thick and stratose 3. S. Miyakei YAMAMOTO

Basidia curved

Probasidial cells clavate.

4. S. clavulatum YAMAMOTO

Probasidial cells subglobose

5. S. bogoriense PATOUILLARD

Basidia without probasidial celis.......... 6. $S$.

Tanakae (MIYABE) BoEdIJN et STEINMANN

\section{Septobasidium nigrum}

YAMAMOTO sp. nov.（桜黑色紋羽菌）

* Laboratory of Plant Pathology, Hyogo University of Agriculture.
Pileo resupinato, effuso, coriaceo, $2 \sim 15 \mathrm{~cm}$. lato, usque $30 \mathrm{~cm}$. longo nigro vel atro-fusco, in sporiferotempore griseo-albo superficie glabro sed margine velutino et determinato, raro totaliter velutino; contextu tristratoso, $290 \sim 530 \mu$ crasso ; strato basilari tenui, $10 \sim 20 \mu$ crasso, ex hyphis fuscidulis, $3 \sim 4 \mu$ diam. constituto; strato medio $170 \sim 440 \mu$ crasso, ex hyphis fuscidulis vel fusco-brunneolis, $3.5 \sim 4.5 \mu$ diam., laxe intertextis et ascendentibus neque columnalibus composito; strato hymeniali $42 \sim 64 \mu$ crasso, ex hyphis densissime ramosis et intertextis, subhyalinis vel hyalinis, $2.5 \sim 4 \mu$ diam. composito; probasidiis globosis, ellipsoideis vel obovoideis, hyalinis, $9.5 \sim 14 \times 5.5 \sim 10.5 \mu$; basidiis subcylindraceis, deorsum leniter attenuatis, rectis, 1 -septatis, hyalinis, $28 \sim 36 \times 5.5 \sim 8 \mu$; sterigmatibus acro-pleurogenis, $10 \sim 35 \mu$ longis; sporis ellipsoideocylindraceis, leniter curvatis, apice obtusis, ad basim apiculatis, hyalinis, $13.5 \sim 24 \times 4 \sim 5.5 \mu$.

Hab. On branches and trunks of Prunus yedoensis (Somei-yoshino). Prov. Tamba: Okano-mura (Dec. 10, 1953, W. Y.); Sasayama (May 14, 1954, W. Y. type); Nishikomume-mura (May 19, 1954, W. Y.). Prov. Settu: Minoo (May 14, 1955, W. Y.) Prov. Kawachi: Kawachi-nagano (May 14, 1955, W. Y.). On Prunus donarium (Sato-zakura). Prov. Tamba: Sasayama (Apr. 18, 1954, W. Y.); Kanegasaka (May 9, 1955, W. Y.); Shino-mura (May 19, 1954, W. Y.). Prov. Yamato: Yoshino-yama (Aug. 8, 1955, W. Y.). On Prunus subhirtella (Higan-zakura). Prov. Settu: Minoo (May 14, 1955, W. Y.).

The present species resembles Septobasidium sinuosum COUCH, but differs from the latter by the smaller size of probasidia, basidia and basidiospores.

\section{Septobasidium tambaensis}

YAMAMOTO sp. nov.（桜暗褐色紋羽菌）

Pileo resupinato, effuso, coriaceo, tenuissimo, $1 \sim$ $4 \mathrm{~cm}$. lato, $1 \sim 7 \mathrm{~cm}$. longo, cinnamomeo-brunneo, superficie glabro, aspero, margine griseo-albo vel albo, determinato; contextu indistincte tristratoso, $180 \sim 340 \mu$ crasso; strato basilari $16 \sim 45 \mu$ crasso, ex hyphis dilute brunneolis, dense lateraliter intertextis, 3 $\sim 4 \mu$ diam. composito; strato medio 95 $244 \mu$ crasso, ex fascicularis hypharum laxe vel dense columnalibus $53 \sim 72 \mu$ altis et $16 \sim 80 \mu$ diam efformato, interdum nec columnalibus efformato, in superiore ex hyphis brunneis, dense oblique vel 

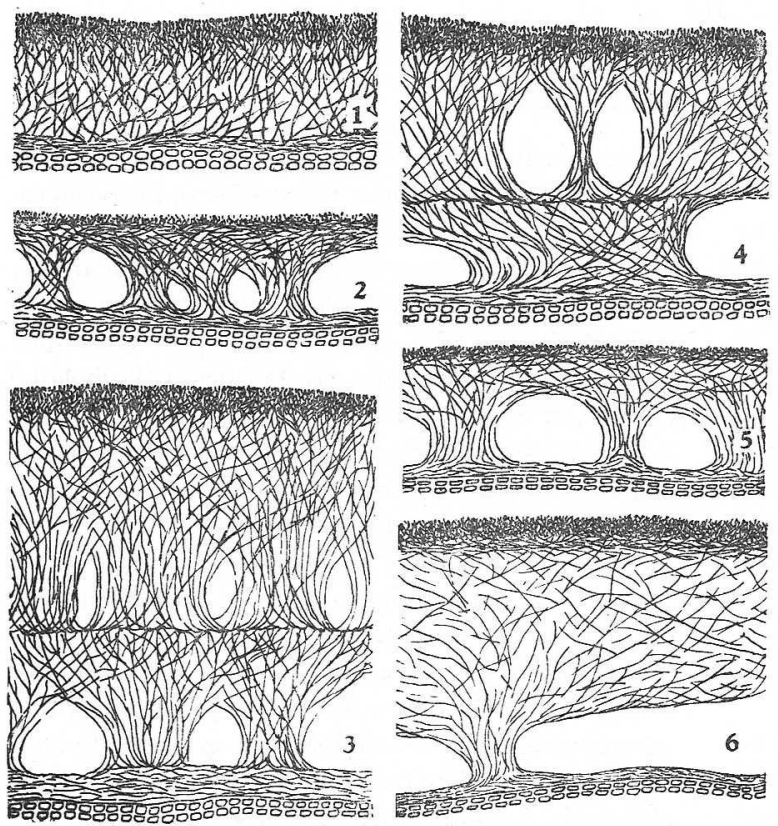

Fig. 1. Septobasiscium nigrum

Fig. 2. Septobasidium tambaensis. Fig. 3. Septoasidium Miyakei. Fig. 4. Septobasidum clavulatum. Fig. 5. Septobasidium bogoriense. Fig. 6. Septobasidium Tanakae. Figs. 1 6, $\times 50$. irregulariter intertextis et ascendentibus composito; strato hymeniali indistincto, $34 \sim 53 \mu$ crasso, ex hyphis hyalinis vel dilute brunneolis, $2.5 \sim 3.5 \mu$ diam. composito; probasidiis subglobosis vel obovoideis, hyalinis, $9 \sim 16 \times 8 \sim 12 \mu$; basidiis subcylindraceis, rectis, apicem versus vel utrimque leniter attenuatis, apice subacutis, 3-septatis, raro ad septa leniter constrictis, hyalinis, $56 \sim 80 \times 5.5 \sim 7.5 \mu$; sterigmatibus pleurogenis, brevibus, $3 \sim 10 \mu$ longis ; sporis ellipsoideo-cylindraceis, leniter curvatis, apice obtusis, ad basim apiculatis, hyalinis, $14 \sim 24 \times 4 \sim$ $5 \mu$.

Hab. On branches and trunks of Prunus donarium var. spontanea (Yama-zakura). Prov. Tamba: Kanegasaka (Jan. 31, 1955; Apr. 26, 1955, W. Y. type).

The present species closely resembles $S$. curtisii (BERK. et DESM.) BOED. et STEINM. in the shape of basidia, sterigmata and basidiospores, but differs from the latter by the structure of top layer and in the color of fructification.

\section{Septobasidium Miyakei}

YAMAMOTO sp.nov。(ぐみとはく色絞羽菌)

Pileo resupinato, effuso, coriaceo, $1 \sim 5 \mathrm{~cm}$. lato, $3 \sim 13 \mathrm{~cm}$. longo, cinnamomeo-brunneo, superficie glabro, rigidulo, margine tenui, pallide brunneo vel albo, determinato; contextu saepe pleuristratoso, $440 \sim 830 \mu$ crasso; strato basilari $25 \sim 55 \mu$ crasso, ex hyphis brunneis, $2.5 \sim 3.5 \mu$ diam., dense lateraliter intertextis composito ; strato medio saepe bi vel tristratoso, $360 \sim 720 \mu$ crasso, ex fascicularis hyparum columnalibus $70 \sim 120 \mu$ altis et $50 \sim 180 \mu$ diam. efformato ; strato hymeniali $45 \sim 60 \mu$ crasso, ex hyphis densissime ramosis et intertextis, pallide brunneolis vel hyalinis, $1.2 \sim 3 \mu$ diam. composito, raro stratoso; probasidiis subglobosis, obovoideis vel piriformibus, hyalinis, 10.5 $19 \times 8 \sim 11 \mu$; basidiis subcylindraceis, rectis, deorsum vel utrimque paulatim attenuatis, apice obtusis vel subacutis, hyalinis, plerumque 3 -septatis, $45 \sim 65 \times 5 \sim 6 \mu$; sterigmatibus pleurogenis, brevibus, $3 \sim 8 \mu$ longis; sporis ellongato-ellipsoideis, leniter curvatis, deorsum leniter attenuatis, apice obtusis, ad basim a piculatis, hyalinis, $12 \sim 19 \times 3 \sim 4 \mu$.

Hab. On branches and trunks of Elaeagnus pungens (Narrashiro-gumi). Prov. Tamba: Johokumura (May 16, 1954, A. MIYAKE, type; Apr. 29, 1955, W. Y.). On Diospyros Kaki (Kaki). Prov. Tamba: Johoku-mura (Jan. 15, 1955, A. MIYAKE).

The present species is closely related to S. ficicolum PAT, but distinguished from the latter by the thicker and stratose middle region.

\section{Septobasidium clavulatum}

YAMAMOTO sp. nov. (ぐみ灰黑色紋羽菌)

Pileo resupinato, effuso, coriaceo, $0.5 \sim 4.5 \mathrm{~cm}$. lato, $1 \sim 15 \mathrm{~cm}$. longo, superficie glabro, atro vel fusco, dein saepe medio pallescento et griseo-albo, interdum submargine griseo-brunneolo, margine albo et determinato, concentriciter zonato; contextu tristratoso, $420 \sim 720 \mu$ crasso; strato basilari $20 \sim$ $40 \mu$ crasso, raro usque $70 \mu$ crasso, ex hyphis fuscobrunneis, lateraliter dense intertextis composito; strato medio $380 \sim 530 \mu$ crasso, interdum bistratoso, ex fascicularis hypharum columnalibus $60 \sim 150 \mu$ altis et $50 \sim 250 \mu$ diam. efformato, in superiore ex hyphis fusco-brunneis, laxe intertextis et ascendentibus, $3 \sim 4 \mu$ diam. composito; strato hymeniali $30 \sim 45 \mu$ crasso, ex hyphis pallide brunneolis vel hyalinis, $2.3 \sim 3.5 \mu$ diam., densissime ramosis et intertextis praedito; probasidiis clavatis, spathulatis, piriformibus vel obovoideis, hyalinis, $14 \sim 32 \times 8.5$ $\sim 12.5 \mu$; basidiis cylindraceis, uncinatis, 3-septatis, hyalinis, $30 \sim 52 \times 6.5 \sim 10 \mu$; sterigmatibus pleurogenis, $10 \sim 25 \mu$ longis ; sporis ellipsoideo-cylindraceis, leniter curvatis, apice obtusis, ad basim apiculatis, hyalinis, $14 \sim 27 \times 3.3 \sim 5.5 \mu$. 


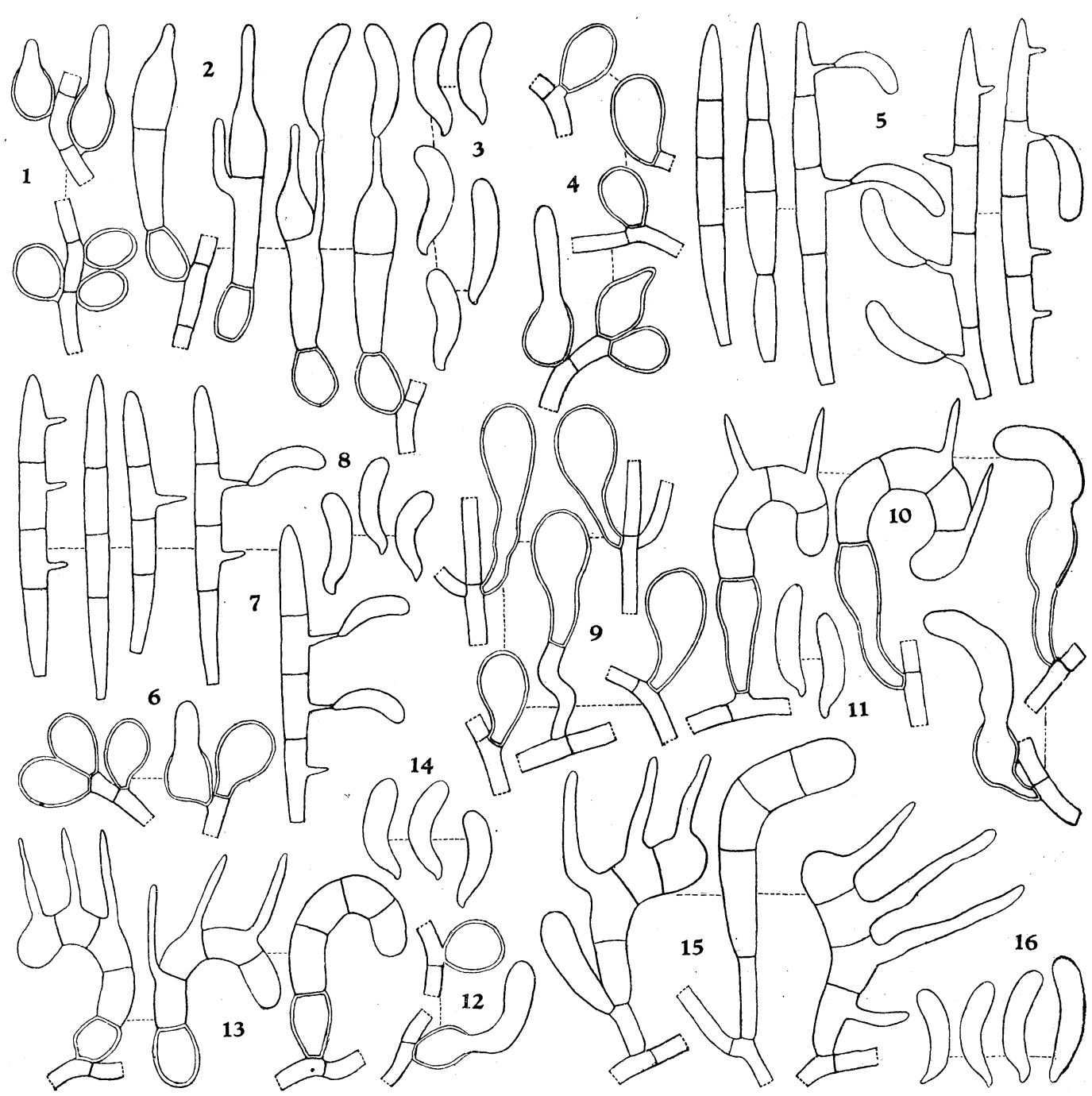

Figs. 1 3. Septobasidium nigrum. 1. Probasidia. 2. Basidia. 3. Basidiospores. Figs. $4 \sim 5$. Septobasidium tambaensis. 4. Probasidia. 5. Basidia and basidiospores. Figs. 6〜8. Septobasidium Miyakei. 6. Probasidia. 7. Basidia. 8. Basidiospores. Figs. 9〜11. Septobasidium clavulatum. 9. Probasidia. 10. Basidia. 11. Basidiospores. Figs. 12 14. Septobasidium bogoriense. 12. Probasidia. 13. Basidia. 14. Basidiospores. Figs. 15 16. Septobasidium Tanakae. $15 . \quad$ Basidia. 16. Basidiospores. Figs. $1 \sim 16 . \times$ ca. 530 .

Hab. On branches and trunks of Elaeagnus multiflora (Natsu-gumi). Prov. Tamba: Johokumura (May 14, 1954, W. "Y. type); Sasayama-cho (Feb. 1, \& Apr. 28, 1955, W. Y.).

The present species resembles $S$. bogoriense PAT. in general character, but differs from the latter by the longer and clavate probasidial cells.

\section{Septobasidium bogoriense}

PATQuillard

in P. Hennings, Monsunia, 1: 138, 1900 ; SACCARDO, Syll. Fung. 16: 184, 1902 ; BOEDIJN
\& STEINMANN, Bull. Jard. Bot. Buitenzorg, III, no. 11: 205, 1931; CoUCH, Genus Septobasidium, 213, 1938; ITO, Myc. Fl. Jap. II, no. 4 : 11, 1955. Septobasidium pedicellatum (non PATOUILLARD) in SAwAdA, Bot. Mag. Tokyo, 26: 307, 1912; Descr. Cat. Form. Fung. 1: 416, 1919; YAsUDA, Bot. Mag. Tokyo, 28 : 477, 1914; FUJIKURO, ibid. 28 : 482, 1914 ; MIYAKE, Bull. Ser. Exp. Sta. I, no. 5: 332, 1916; Lloyd, Lett. 46: 7, 1917.

Septobasidium Mompa (TANAKA) RADIBORSKI in Bull. Acad. Sci. Cracovie, 3: 355, 1909; SACCARDO, 1. c., $21: 445,1912$; COUCH, Genus 


\section{Septobasieium, 213, 1938.}

\section{Septobasidium indigophorum $\mathrm{COUCH}$}

Genus Septobasidium, 282, 1938; ITO, Myc. Fl. Jap. II, no. 4: 14, 1955.

Fructification resupinate, effused, coriaceous, 1.5 $\sim 7 \mathrm{~cm}$. long, $1 \sim 5 \mathrm{~cm}$. wide. Surface inherently smooth, lilac gray, purpulish dark gray to purpulish black. Margin determinate, fibrillose and white. Context usually composed of three layers, in section $200 \sim 480 \mu$ thick. Subiculum thin, usually $15 \sim 30 \mu$ thick, composed of pale brownish to dark brownish hyphae which are $3 \sim 4 \mu$ thick. From the subiculum arise short, stubby pillars, $40 \sim 130 \mu$ thick by $55 \sim 125 \mu$ tall. The hyphae of the pillars loosely arranged, ascending, dark brownish, $3 \sim 4 \mu$ thick. Hymenial layer $30 \sim 65 \mu$ thick, composed of densely interwoven, hyaline to brownich hyphae which are $2.3 \sim 3 \mu$ thick. Probasidia subglobose, $8 \sim 12$ $\times 6.5 \sim 11 \mu$. Basidia cylindric, curved, 3-septate, $29 \sim 40 \times 5.5 \sim 8 \mu$. Sterigmata pleurogeǹous, $7 \sim 20 \mu$ long. Basidiospores long elliptic, slightly curved, apiculate at the basal end, hyaline, $12 \sim 24 \times 3.5 \sim$ $5.5 \mu$.

Hab. On branches and trunks of Broussonetia Kazinoki SIEB. (Kozo). Prov. Tamba: Okanomura (W. Y.). On B. papyrifera (Kazinoki). Prov. Tamba: Okano-mura (W. Y.). On Mallotus japonicus (Akame-gashiwa). Prov. Tamba: Okanomura (W. Y.). On Morus bombycis (Kuwa). Prov. Tamba: Okano-mura (A. MIYAKE); Johoku-mura (W. Y.). On Paulownia tomentosa (Kiri). Prov. Tamba: Okano-mura (W. Y.). On Xanthoxylum piperitum (Sansho). Prov. Tamba: Okano-mura (W. Y.); Maki-mura (W. Y.).

The present species has generally been referred to $S$. pedicellatum in Japan, but was established by ITO (1955) that the fungus is identical with $S$. bogoriense PAT. S. indigophorum COUCH on the flowering cherry trees which was originally collected at Shino-mura, Prov. Tamba and described as new species but it seems to be not disfinct from $\mathrm{S}$. bogoriense.

\section{Septobasidium Tanakae \\ (Miyabe) Boedijn et Steinmnn}

in Bull. Jard. Bot. Buitenzorg, III, no. 11: 169, 1931 ; ITO, Myc. Fl. Jap. II, no. 4: 13, 1955.

Helicobasidium Tanakae MIYABE in SAWADA, Bot. Mag. Tokyo, 26: 104 \& 307, 1912; Descr. Cat. Form. Fung. II : 104, 1922; MIYAKE, Bull. Ser. Exp. Sta. I, no. 5: 333, 1916; SACCARDO, l. c. $23: 558,1925$.

Septobasidium prunophilum COUCH

Genus Septobasidium, 280, 1938; ITO, Myc. Fl.
Jap. II, no. 4: 14, 1955.

Fructification resupinate, effused, coriaceous, $1 \sim$ $9 \mathrm{~cm}$. long, $0.8 \sim 8 \mathrm{~cm}$. wide. Surface velvety, deep brown. Margin determinate, finely fimbriate, grayish white to white. Context usually composed of three layers; in section $300 \sim 520 \mu$ thick. Subiculum thin, dark brown, compact, $15 \sim 30 \mu$ thick, from the subiculum arise the rather scattered, short and stubby pillars, $40 \sim 170 \mu$ thick by $40 \sim 138 \mu$ tall. The hyphae of the pillars deep brown, 3.5 $\sim 5 \mu$ thick, branching out above to the hymenial layer. Hymenial layer 53 96 $\mu$ thick, composed of brownish to hyaline, upright, branched, straight or wavy, closely arranged hyphae which are 3 $4 \mu$ thick. Probasidia not formed. Basidia formed directly from the hyphae in the basal region of hymenium, clavate, more or less curved, rounded at apex, 3-septate, $27 \sim 53 \times 8 \sim 11 \mu$. Sterigmata pleurogenous, $20 \sim 45 \mu$ long. Basidiospores long elliptic, slightly curved, obtuse at the apex, apiculate at the basal end, hyaline, $16 \sim 29 \times 4 \sim 6.5 \mu$.

Hab. On branches and trunks of Broussonetia Kazinoki (Kozo). Prov. Tamba: Okano-mura (W. Y.). On Diospyros Kaki (Kaki). Prov. Tamba: Sasayama (W. Y.). On Morus bombycis (Kuwa). Prov. Tamba: Okano-mura (A. MIYAKE); Johokumura (W. Y.). On Osmanthus fragrans var. aurantiacus (Kin-mokusei). Prov. Tamba: Sasayama (W. Y.). On Paulownia tomentosa (Kiri). Prov. Tamba: Okano-mura (W. Y.). On Prunus Armeniaca var. Ansu (Anzu). Prov. Tamba: Sasayama (A. MIYAKE). On $P$. donarium Sieb. var. spontanea MAK. (Yama-zakura). Prov. Tamba: Kanegasaka (W. Y.). On P. Mume (Ume). Prov. Tamba: Sasayama (W. Y.); Okano-mura (A. MIYAKE); Maki-mura (W. Y.); Shino-mura (W. Y.). Prov. Kawachi: Kawachi-nagano (W. Y.). On P. Persica (Momo). Prov. Tamba: Sasayama (W. Y.). On P. salicina (Sumomo). Prov. Tamba: Sasayama (W. Y.). Prov. Settu: Minoo (W. Y.). On $P$. yedoensis (Somei-yoshino). Prov. Tamba: Sasayama (W. Y.); Okano-mura (A. MIYAKE); Shino-mura (W. Y.). Prov. Yamashiro: Arashiyama (W. Y.). On Xanthoxylum piperitum (Sansho). Prov. Tamba: Okano-mura (A. MIYAKE); Sasayama (A. MIYAKe). Prov. Yamato: Yoshinoyama (W. Y.)

Although the present species has generally been referred to Helicobasidium Tanakae MIYABE in Japan, it was tranfered to Septobasidium by BOEDIJN and STEINMANN (1931). S. prunophilum Couch which was originally collected at Shinomura, Prov. Tamba seems to be identical with the present species. (Received August 18, 1955) 